D the discovery was pronounced a mirage. The final straw came with an experiment at the Thomas Jefferson National Accelerator Facility in Newport News, Virginia, that repeated the SPring-8 measurement with more data and ruled out the pentaquark's existence.

The episode has been held up as an example of how scientists can be tricked by data into seeing more than is there. In 2008, the annual Review of Particle Physics ${ }^{1}$ described the pentaquark as "a curious episode in the history of science", and its recent listings have no dedicated entry for the particle.

But the LHCb result leaves little doubt that pentaquarks are real, researchers say. Physicists saw a signal showing the unexpected appearance of two short-lived objects weighing 4.38 and 4.45 gigaelectronvolts (4.67 and 4.74 times heavier than a proton) during the decay of trillions of subatomic particles known as 'Lambda B' baryons, by analysing data from 2009-12.
Artist's impression of a pentaquark.

After exhausting other known particles as candidates, the team concluded that the new objects correspond to a pentaquark in two different configurations. The particle contains two 'up' quarks, a 'down' quark, and a 'charm' quark-antiquark pair, making it a 'charmonium' pentaquark. A preprint on the find was posted on the arXiv server on $14 \mathrm{July}^{2}$, and has been submitted for publication in Physics Review Letters. "It's about the most exciting discovery in QCD I could imagine," says Frank Wilczek, one of the original architects of QCD, at the Massachusetts Institute of Technology.

The result, which first caught the attention of physicists on LHCb in 2012 as a bump in their data, was a total surprise, says LHCb's Sheldon Stone, at Syracuse University in New York. "In the old days we searched for new particles by bump-hunting, but in this case the bump found us!" he says. "For historical reasons we were quite haunted by the word pentaquark, so we did every conceivable check we could," he says. The LHCb team says that there is a vanishingly small chance of the signal appearing if no new particles existed. Their statistical bar - known as 9-sigma - is higher than the 5-sigma usually required for a discovery in particle physics.

"If I have an immediate feeling of worry it is that they claim two states: is this because they have found a process that favours production of pentaquarks, or because they have not really found the best interpretation of the data?" says theorist Frank Close, of the University of Oxford, UK.

The new pentaquark is not the one, known as the theta+, seen back in 2002: it is almost three times heavier, and contains different kinds of quarks. "I think our result will energize the search for many different pentaquark states, including the debunked theta+," says Stone.

1. Particle Data Group. Phys. Lett. B 667, 1061-1206 (2008).

2. LHCb collaboration. Preprint at http://arxiv.org/ abs/1507.03414 (2015).

\title{
First robust genetic links to depression emerge
}

\section{Discoveries energize hunt for genes connected to mental illness.}

\section{BY HEIDI LEDFORD}

$\mathrm{N}$ o one was more surprised than Jonathan Flint when his project - an effort to find genetic sequences linked to depression - showed the first hint of success 18 months ago. He knew the odds were slim: a study of 9,000 people with major depressive disorder had come up empty ${ }^{1}$, and Flint had heard rumours that a follow-up analysis of 17,000 people had also met with disappointment. "I thought, 'There's no way," says the geneticist from the University of Oxford, UK, whose study had by that point analysed only 5,303 people with depression.

Flint has proved himself wrong. In Nature this week, his team reports ${ }^{2}$ the first two genetic markers reproducibly linked to major depressive disorder, one of the leading causes of disability globally. The findings could guide biologists to new drugs, and could one day be used to aid diagnosis. But many in the field are excited that the markers have been unearthed at all. The results look set to end years of debate over whether sequences for such a complex disorder could be found - and Flint's study may serve as a framework for future attempts to collect data from tens of thousands of people.

More than 350 million people have depression. The disorder's symptoms and severity can vary widely from one person to the next, and particularly between men and women. This suggests that different conditions have been lumped together into one diagnosis, complicating genetic analyses (see Nature 515, 182-184; 2014)

The few hits from early studies attempting to find genetic sequences linked to depression had disappeared on closer scrutiny, so Flint knew that he would need samples from thousands of people, and a way to reduce the variability in their illness. Flint and Kenneth Kindler, a psychiatrist at Virginia Commonwealth University in Richmond who is renowned for his diagnostic prowess, decided to do the study in China, because of its large population and because depression is believed to be under-diagnosed there. In that climate, Flint reasoned, those who are diagnosed are likely to share a severe form of the disorder. To reduce the variability further, his team also limited the study to women of Han Chinese ethnicity.

By early 2014, Flint, Kindler and a team of collaborators had analysed DNA sequences from 5,303 Chinese women with depression, and another 5,337 controls. As Flint expected, $85 \%$ of the depressed women had a severe form of the disorder called melancholia, which robs people of the ability to feel joy. "You can be a doting grandparent and your favourite grandchildren can show up at your door," says Douglas Levinson, a psychiatrist at Stanford University in California, "and you can't feel anything."

The analysis yielded two genetic sequences that seemed to be linked to depression: one in a stretch of DNA that codes for an enzyme whose function is not fully understood, and the other next to the gene SIRT1, which is important for energy-producing cell structures called mitochondria. The correlations were confirmed in another set of more than 3,000 depressed men and women and over 3,000 controls.

The mitochondrial connection chimes with previous work, including some from Flint's lab ${ }^{3}$, that has linked mitochondrial abnormalities to 


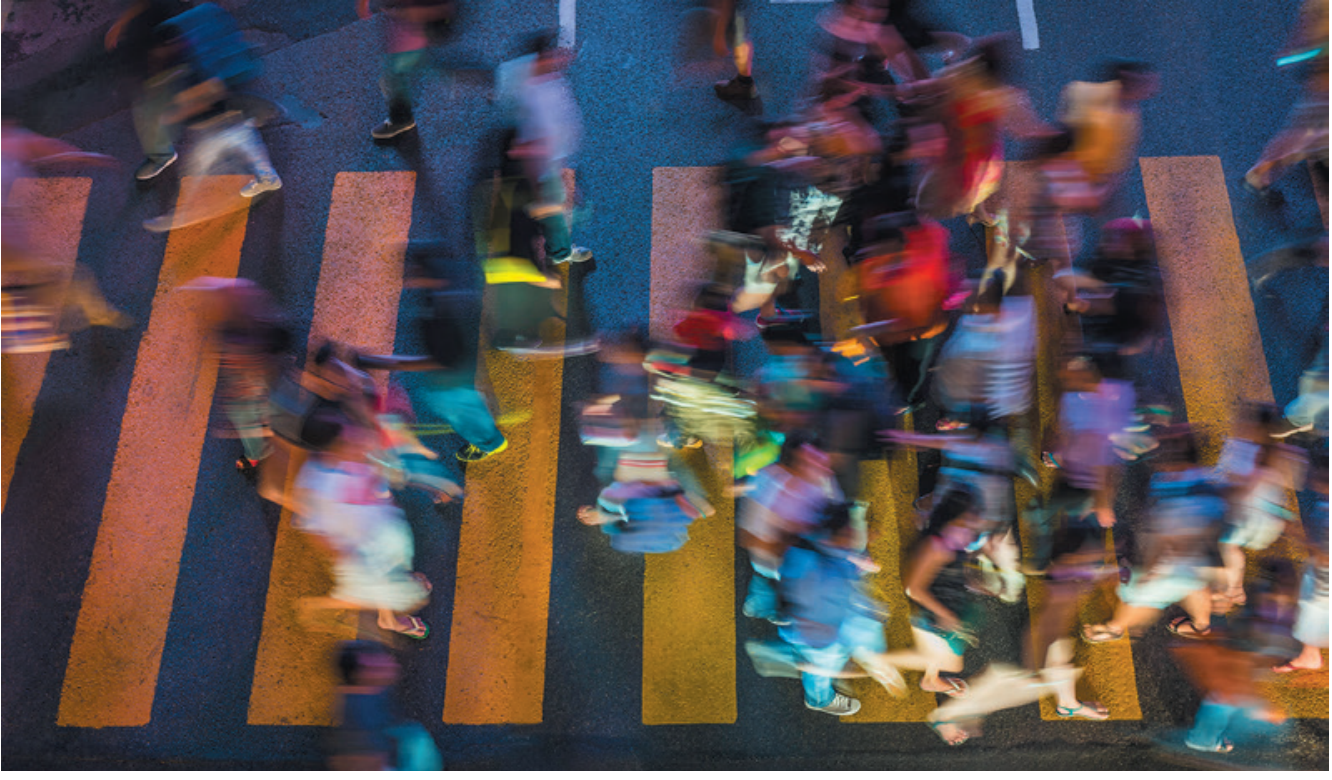

Depression can take many forms, making it hard to tease out genetic links.

depression. "It's an appealing bit of biology for ज a disorder that makes people tired and unmotivated," says Levinson.

But he is most intrigued by the way Flint and his colleagues designed their study. Levinson of says that the Psychiatric Genomics Consortium, of which he is a member, has analysed data from 17,000 people with depression without finding a genetic hit, as Flint had heard. In light of Flint's results, the group, which is made up of researchers who agree to pool genetic data, is now investigating whether limiting the analysis to people with particularly severe depression might change things.

If it does, still more samples will be needed before other genetic links emerge. Levinson expects a series of biobanking efforts in places including the United Kingdom, the Netherlands and Australia to provide tens of thousands of genomes for analysis within the next five years. The hope is that as more genetic links are found, they will flag up groups of proteins known to work together to affect certain cellular functions: these 'pathways' could be investigated as drug targets, and for their potential to make diagnosis of depression more definitive.
Flint's success may energize that search, says Patrick Sullivan, a psychiatric geneticist at the University of North Carolina at Chapel Hill. "We've had to learn not to listen a lot to our critics," he says. "If we listened to people telling us that what we're doing is stupid, we would have stopped years ago."

1. Major Depressive Disorder Working Group of the Psychiatric GWAS Consortium Mol. Psychiatry 18, 497-511 (2013).

2. CONVERGE Consortium Nature http://dx.doi. org/10.1038/nature14659 (2015).

3. Cai, N. et al. Curr. Biol. 25, 1146-1156 (2015).

\section{CORRECTIONS}

The News Feature 'Weighing the world's trees' (Nature 523, 20-22; 2015)

incorrectly described the photosynthesis measurements made by the Orbiting Carbon Observatory-2. Those observations are made by the same spectrometers that measure $\mathrm{CO}_{2}$ concentrations.

The News story 'Researchers pin down risks of low-dose radiation' (Nature 523, 17-18; 2015) incorrectly calculated an 'expected' death rate from leukaemia among the workers. Only 30 deaths were attributable to the radiation, and the relative risk increment posed by each additional $10 \mathrm{mSv}$ was $3 \%$. 